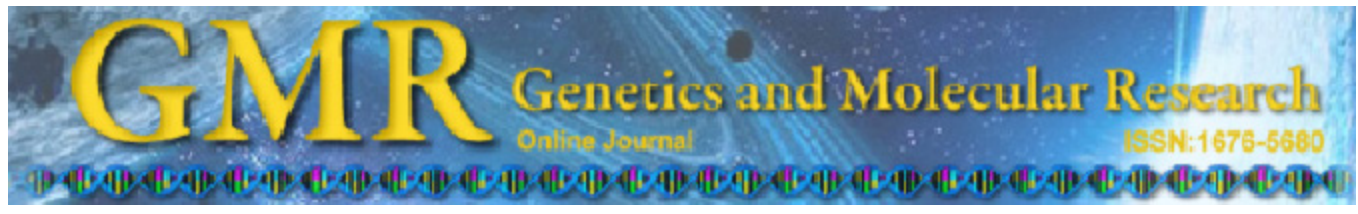

\title{
Clinical three-dimensional conformal radiotherapy research using repeated computed tomography scans for field reduction in older non-small-cell lung cancer patients
}

\author{
Z.Z. Fu ${ }^{1 *}$, T. Gu${ }^{1 *}$, S.H. Zhang ${ }^{2}$, X.Y. Cao ${ }^{2}$, Q.H. Zhang ${ }^{1}$, Y.Q. Zhang ${ }^{1}$, \\ H.X. Hua ${ }^{1}$, S. Yang ${ }^{1}$, P. Li ${ }^{1}$ and Z. Zhang ${ }^{2}$ \\ ${ }^{1}$ Department of Oncology, The First Hospital of Qinhuangdao, Qinhuangdao, \\ China \\ ${ }^{2}$ Department of Oncology, Tangshan Workers Hospital, Tangshan, China \\ *These authors contributed equally to this study. \\ Corresponding author: T. Gu \\ E-mail: taogucn@yeah.net
}

Genet. Mol. Res. 13 (3): 5343-5349 (2014)

Received May 27, 2013

Accepted November 12, 2013

Published July 24, 2014

DOI http://dx.doi.org/10.4238/2014.July.24.13

\begin{abstract}
This study investigated the curative and toxic effects of three-dimensional conformal radiotherapy (3D-CRT), using repeated CT scans for field reduction in older non-small-cell lung cancer (NSCLC) patients. 3D-CRT was administered to 36 older patients with NSCLC, and irradiation fields included the primary lesion and metastatic lymph nodes. After CT localization scanning, images were fed into a treatment planning system to delineate the gross tumor volume (GTV)1 and prepare Plan 1. After the DT50 (dose of the tumor is 50 Gy) increased from 50 Gy in 25 fractions to 54 Gy in 27 fractions, secondary CT localization scanning was performed to delineate GTV2 and prepare Plan 2; radiotherapy was administered continuously. When the DT
\end{abstract}


increased to 60-65 Gy, tertiary CT scanning was performed to prepare another plan. The field was reduced to boost irradiation to the residual target volume until the total DT increased to 68-74 Gy. Compared with GTV1, the median absolute volume regression and median relative regression amounts for GTV2 were $68.85 \mathrm{~cm}^{3}$ and $31.17 \%$, respectively $(Z=-2.673, P=0.021)$. There were 8 cases of complete remission (22.2\%), 20 of partial remission (55.6\%), 7 of stable disease (19.4\%), and 1 of progressive disease (2.8\%). The total effectiveness rate was $77.8 \%$ and the 1- and 2-year survival rates were 63.9 and $27.8 \%$, respectively. Radiation esophagitis and radiation pneumonia, the main toxic side effects, were tolerable. 3D-CRT, using repeated CT scans for field reduction in older NSCLC patients, could increase the local control and survival rates and relieve the toxic radiotherapy side effects.

Key words: Non-small-cell lung cancer; Repeated CT scans; Three-dimensional conformal radiotherapy; Field reduction increment; Older patient; Treatment result

\section{INTRODUCTION}

Currently, the problem of population aging is becoming increasingly apparent, and the numbers of older patients with lung cancer are increasing. In the USA, it was reported that two-thirds of lung cancer patients were older than 65 years of age, and the incidence rate in people older than 65 years of age was 9.8 -fold higher than that of people younger than 65 years; additionally, the mortality rate of lung cancer patients older than 65 years of age was 16.5-fold higher than that patients younger than 65 years (O'Brien et al., 2003). This problem should be carefully considered by radiation oncology physicians when preparing radiotherapy plans for older lung cancer patients. Currently, radiotherapy plans are generally designed on the basis of tumor imaging, taken at a single time point before radiotherapy. However, during lung cancer radiotherapy, the tumor and surrounding normal tissues exhibit temporal and spatial changes in response to each treatment and between the various treatments. Some studies have suggested that during radiotherapy for non-small-cell lung cancer (NSCLC), the gross tumor volume (GTV) apparently changes. Fox et al. (2009) investigated 22 cases of stage I to III NSCLC patients who received conventional fractionated radiotherapy. Among them, 15 cases received synchronous chemoradiotherapy. When the radiotherapy doses were increased to 30-50 Gy, secondary and tertiary computed tomography (CT) localization scans were performed. Compared with the initial CT localization, the median GTV of the secondary localization was reduced by $24.7 \%$ (range $=-0.3-61.7 \% ; \mathrm{P}<0.001$ ), and the median GTV of the tertiary localization was reduced by $44.3 \%$ (range $=0.2-81.6 \% ; \mathrm{P}<0.001$ ). Woodford et al. (2007) evaluated 17 NSCLC patients and found that after 30 cycles of conventional fractionated radiotherapy, the GTV was reduced by an average of $38 \%$ (range $=12-87 \%$ ).

However, there is no current and uniform viewpoint on the extent of influence that a change in the radiotherapy target area would have on treatment plans and the opportunities to modify treatment plans. In radiotherapy, positron emission tomography-CT, on-line or 
off-line CT, cone-beam CT image analysis, and other novel techniques provide possibilities for self-adaptive radiotherapy. This study used repeated CT scanning to determine changes in tumor volumes and morphologies in order to analyze the basic change rule for tumor volumes in radiotherapy treatment courses. Concomitantly, this study analyzed the curative and toxic side effects of radiotherapy and conducted a preliminary investigation of its application value.

\section{MATERIAL AND METHODS}

\section{General data}

This study evaluated 36 older NSCLC patients who were treated at the Oncology Department of Qinhuangdao First Hospital and the Oncology Department of Tangshan City Workers' Hospital between October 2006 and December 2008, including 25 male and $11 \mathrm{fe}-$ male patients. The patient age at treatment ranged from 71 to 78 years old, and the median age was 75 years old. After lung cancer surgery, 5 cases presented tumor recurrences. Additionally, a pathologic examination confirmed that there were 23 squamous cell carcinoma and 13 adenocarcinoma cases. Before treatment, all patients underwent comprehensive physical examinations, including routine blood analyses; heart, liver, and kidney examinations; and chest CT scans. Additionally, the Karnofsky score of the overall group exceeded 70. This study was conducted in accordance with the Declaration of Helsinki and with the approval of the Ethics Committee of the First Hospital of Qinhuangdao City. Written informed consent was obtained from all participants.

\section{Radiotherapy method}

Each patient rested in the supine position and propped his/her head on both upper arms, at which point the body position was fixed with thermoplastic film. A Prospeed S Fast CT (GE Company, Fairfield, CT, USA) was used for the scans. The interlayer spacing was 5 $\mathrm{mm}$, and the planned scanning range was from the thoracic entrance to the costophrenic angle. At the end of localization scanning, the CT image information was transmitted and converted with the VENUS 3-D treatment plan system computer workstation (Shanghai Topslane Company, Shanghai, China). Subsequently, 2 radiotherapy physicians delineated the target volumes layer-by-layer and completed 3-D reconstructions of the anatomical images. According to the CT-scanned pulmonary images, the GTV1 was delineated to include the primary tumor and regional lymph node metastases $(>1.0 \mathrm{~cm}$ or $>3$ lymph node bundles). The clinical target volume (CTV)1 was set at 6-8 $\mathrm{mm}$ outside of the GTV, and the planning target volume (PTV)1 was set at 5-15 mm outside of the CTV. Additionally, 4-6 fixed fields or non-coplanar fields were used to cover the PTV1 with the $90-95 \%$ isodose curve. Simultaneously, the PRIMUS Type linear accelerator 6MV-X (SIEMENS Company, Munich, Germany) was used to administer a DT (dose of the tumor) regimen of $2.0 \mathrm{~Gy} /$ fraction at 1 fraction/day, 5 days/week. When the DT ranged from 50-54 Gy, a secondary CT localization scan was performed to obtain the CT2. After the scans were processed according to the requirements, the CTV2 and PTV2 were obtained and radiotherapy was continuously administered in a DT regimen of 2.5-3.0 Gy/fraction at 1 fraction/day, 5 days/week. When the DT ranged from 60-65 Gy, a tertiary CT scan was performed in the same manner. The field was reduced to boost the irradiation dose to the 
residual target volume (DT of 2.5-3.0 Gy/fraction, 1 fraction/day, 5 days/weeks) until the total DT ranged from 68-74 Gy. Dose volume histograms and isodose curves were used to comprehensively evaluate the treatment plans. The optimization indicators included the percent of PTV that received the prescribed dose. The PTV uniformity was $\pm 5 \%$ of the prescribed dose, the maximum doses to important organs outside the PTV did not exceed the prescribed dose, and the doses to key organs did not exceed the tolerance doses of normal tissues (total lung dose and contralateral lung).

\section{Assessment of curative effects and toxic side effects}

According to the World Health Organization (WHO) reporting standard on curative effects in tumors, the curative effects were divided into complete remission (CR), partial remission (PR), stable disease (SD), and progressive disease (PD) groups, and the total effectiveness rate was calculated as (CR + PR) x 100\%. Assessments were performed according to chest CT scans, and the curative effects were jointly determined by 2 experienced radiologists. Additionally, evaluations of acute and chronic toxicity and side effects included the lungs, esophagus, spinal cord, and heart. The assessment criteria respectively complied with the Radiation Therapy Oncology Group (RTOG) criteria and the RTOG/European Organisation for Research and Treatment of Cancer criteria.

\section{Statistical analysis} tions.

The SPSS version 11.5 software (SSPS Inc., Chicago, IL, USA) was used for calcula-

\section{RESULTS}

\section{Changes in tumor volumes}

The tumors regressed during radiotherapy. The median GTV1 was $218.02 \mathrm{~cm}^{3}$ $\left(\right.$ range $\left.=129.68-366.56 \mathrm{~cm}^{3}\right)$, and the median GTV2 was $149.10 \mathrm{~cm}^{3}($ range $=85.47-287.15$ $\left.\mathrm{cm}^{3}\right)$. There was a significant difference between the 2 volumes $(\mathrm{Z}=-2.673, \mathrm{P}=0.021)$. Compared with the GTV1, the absolute volume regression amount of GTV2 was $68.85 \mathrm{~cm}^{3}$ and the median relative regression amount was $31.17 \%$. Additionally, the mean regression rate was $0.93 \% /$ day.

\section{Recent curative effects and reactions}

All patients completed radiotherapy according to the treatment plan, and there were 8 cases of CR (22.2\%), 20 cases of PR (55.6\%), 7 cases of SD (19.4\%), and 1 case of PD (2.8\%). The total effective rate $(\mathrm{CR}+\mathrm{PR})$ was $77.8 \%$. Additionally, the incidence rates of grade $\mathrm{I} / \mathrm{II}$ and grade III acute radiation esophagitis were 50.0\% (18/36) and 5.6\% (2/36), respectively, with no grade IV cases. As a result, these patients recovered after symptomatic treatment. Additionally, the incidence rates of grade I/II and grade III acute radiation pneumonitis were $13.9 \%(5 / 36)$ and $5.6 \%(2 / 36)$, respectively, with no grade IV cases. There were no heart or spinal cord reactions. 


\section{Follow-up and survival situations}

For all cases, the follow-up date was calculated from the end of treatment to October 1,2011 , and the follow-up time ranged from 5-32 months. Additionally, the follow-up rate was $100 \%$, and the overall median survival time was 13.5 months. For 23 cases, the survival duration exceeded 1 year, and the 1 -year survival rate was $63.9 \%$. For 10 cases, the survival duration exceeded 2 years, and the 2-year survival rate was $27.8 \%$. Among the patients, 15 died of extensive tumor metastasis, 10 died of pulmonary infection and respiratory failure, and 4 died of cardiovascular, cerebrovascular, and other diseases.

\section{DISCUSSION}

Currently, society is aging and gerontology research is of gradually increasing concern. Statistical analyses show that the incidence rate of older people with lung cancer is increasing along with the population age. A large body of clinical and basic research data suggest that the application of 3D-conformal radiotherapy (CRT) technique could adjust the highenergy beam shape and permit the distribution of ideal ray doses in human tissues from a ray volume, with a uniform dose distribution similar to that of the 3-D spatial volume and shape in the target area, thus helping to reduce the irradiation doses to normal tissues and increase the target dose (Grills et al., 2007). Tumor radiotherapy precision is among the main factors that influence the curative effect on NSCLC. The NSCLC volume and location change between fractionated radiotherapy doses, and these changes can induce corresponding modifications to the radiotherapy plan through self-adaptive radiotherapy (Guckenberger et al., 2011). In cases with limited resources, repeated CT localization scanning during radiotherapy is an effective way to monitor target area changes and modify radiotherapy plans.

The tumor volume regression extent varies widely among different individuals. However, no individual tumor or patient factor related to tumor volume change is currently known. Feng et al. (2009) thought that the GTV would change after the radiotherapy dose was increased to 40-50 Gy, and in cases for which the radiotherapy dose was $58 \mathrm{~Gy}$, the average GTV reduction was $26 \%$ (range $=+15$ to $-75 \%$ ). Erridge et al. (2003) used an electronic portal imaging device to evaluate 25 patients and determined that $40 \%$ patients had tumor volume reductions that exceeded 20\% during radiotherapy, while Britton et al. (2007) observed that during radiotherapy, the primary tumor volume reductions in $50 \%$ of the patients exceeded $40 \%$. van Zwienen et al. (2008) evaluated 114 lung cancer patients; among them, $40 \%$ of the patients presented significant tumor regression, while the tumor volumes of $8 \%$ of the patients decreased by $25 \%$ in the third week and more than $17 \%$ in the fourth week. During radiotherapy, the patients in this group of the current study presented a range of tumor regression percentages, and the mean relative regression rate was $0.93 \%$ /day. This result was slightly lower than the rate of $1.2 \%$ / day reported by Kupelian et al. (2005); this difference could possibly be attributed to the fact that there were fewer cases in the current study group and all were older lung cancer patients, which possibly is associated with the pathological type. However, Siker et al. (2006) found no apparent correlations between tumor regression and chemotherapy, pathology, and the original tumor volume. The tumor regression rate had no important correlations with the original tumor volume, radiotherapy duration, pathological type and stage, and tumor density; specifically, the change in primary lesion tumor volume did not correlate with either patient-related properties or tumor-intrinsic characteristics (Woodford et al., 2007). 
A DT of 50-54 Gy was selected for scanning because, with this regimen, subclinical lesions had received adequate doses. During this regimen, the target area is modified to prepare the treatment plan. Another 3D-CRT plan is then prepared according to the reduced tumor volume, which not only safely increases the target dose, but also has an important impact on irradiation dose reductions to normal tissue and organ volumes. Earlier radiotherapy plan modification was not found to be better. One reason is that the premature reduction of irradiation target area could possibly cause insufficient doses to partial subclinical lesions and thus become the source of tumor recurrence or metastasis. Additionally, the tumor volume shows no apparent change upon repeated localizations, it is not feasible to effectively reduce the dose volume parameter of the normal lung tissues. Therefore, there is no significant association with an increased irradiation dose (Woodford et al., 2007). The key to effectively reducing the dose volume parameter is to modify the treatment plan in a timely manner as the tumor volume shows an apparent change.

Although this research method has some limitations such as bad image quality, a smaller sample size, and heterogeneity in treatment modes and doses, which could influence tumor regression, currently the factors related to changes in tumor volume during radiotherapy remain completely unclear.

Clinical dosimetry studies have shown that the irradiation dose is positively associated with the local control rate; namely, the higher the dose, the higher the local control rate. Therefore, an increased target dose is a key factor in the treatment of locally advanced NSCLC. According to the results of the RTOG9701 study (Perez et al., 1980), NSCLC patients who received doses of 40, 50, and 60 Gy had remission rates (including CR + PR) of 48, 53, and $56 \%$, respectively. Sim et al. (2001) reported the conformal radiotherapy results of 152 cases of locally advanced NSCLC. Among them, 70 cases received simple conformal radiotherapy. The median survival duration was 11.7 months and the 1- and 2-year survival rates were 47 and $23 \%$, respectively. Hansen et al. (2005) reported the results of 158 stage III cases that underwent 3D-CRT. Among them, 77 cases received new adjuvant chemotherapy, and the results showed a median survival duration of 15.8 months and 1- and 2-year survival rates of 61 and 35\%, respectively. Armstrong et al. (1997) reported 45 cases of NSCLC. Among them, $89 \%$ were locally advanced cases, while the other cases were stage I/II. The overall survival duration was 15.7 months, and the incidence rate of grade 3 or higher pulmonary toxicity was $9 \%$. In the current study, the curative effect was similar to the results of the above-mentioned reports, but the toxic side effects were apparently reduced.

The influence of accelerated tumor repopulation on the local tumor control rate is of increasing concern. A reduction in the total radiotherapy treatment course can reduce the required time and opportunity for accelerated tumor cell repopulation. In cases with accelerated tumor cell repopulation, the administration of doses higher than those used in conventional radiotherapy can inhibit tumor cell repopulation (Wu et al., 2003). Chen et al. (2000) analyzed the correlation between the NSCLC radiotherapy treatment course and the curative effect and confirmed that extending the total radiotherapy treatment course could decrease the local tumor control rate, thus confirming that reducing the treatment course duration can enhance the curative effect of NSCLC radiotherapy. Currently, there is no definite conclusion regarding the time at which tumor cells undergo accelerated repopulation during the NSCLC radiotherapy process. Given that the tumor epithelial origins are the same, it is speculated that accelerated NSCLC repopulation possibly occurs during medium-to-late conventional radiotherapy treatment courses. Therefore, the fractioned irradiation method of 2.5-3.0 Gy/day is used to administer higher-dose irradiation for a short time after conventional radiotherapy ( 5 weeks) and 
achieve increased local tumor control by increasing the total irradiation dose and fractioned dose and reducing the total treatment course to improving the curative effect. In this study, the total effectiveness rate reached $77.8 \%$, which agreed with the result reported by Wang et al. (2006), while the incidence rates of acute radiation esophagitis and acute radiation pneumonitis were lower than those reported in the relevant literature, indicating a satisfactory recent curative effect of repeated-location 3D-CRT for field reduction in older NSCLC patients, with tolerable early radiotherapy reactions. However, pulmonary infection remains an important threat, according to cause-of-death analyses.

\section{REFERENCES}

Armstrong J, Raben A, Zelefsky M, Burt M, et al. (1997). Promising survival with three-dimensional conformal radiation therapy for non-small cell lung cancer. Radiother. Oncol. 44: 17-22.

Britton KR, Starkschall G, Tucker SL, Pan T, et al. (2007). Assessment of gross tumor volume regression and motion changes during radiotherapy for non-small-cell lung cancer as measured by four-dimensional computed tomography. Int. J. Radiat. Oncol. Biol. Phys. 68: 1036-1046.

Chen M, Jiang GL, Fu XL, Wang LJ, et al. (2000). The impact of overall treatment time on outcomes in radiation therapy for non-small cell lung cancer. Lung Cancer 28: 11-19.

Erridge SC, Seppenwoolde Y, Muller SH, van Herk M, et al. (2003). Portal imaging to assess set-up errors, tumor motion and tumor shrinkage during conformal radiotherapy of non-small cell lung cancer. Radiother. Oncol. 66: 75-85.

Feng M, Kong FM, Gross M, Fernando S, et al. (2009). Using fluorodeoxyglucose positron emission tomography to assess tumor volume during radiotherapy for non-small-cell lung cancer and its potential impact on adaptive dose escalation and normal tissue sparing. Int. J. Radiat. Oncol. Biol. Phys. 73: 1228-1234.

Fox J, Ford E, Redmond K, Zhou J, et al. (2009). Quantification of tumor volume changes during radiotherapy for nonsmall-cell lung cancer. Int. J. Radiat. Oncol. Biol. Phys. 74: 341-348.

Grills IS, Yan D, Black QC, Wong CY, et al. (2007). Clinical implications of defining the gross tumor volume with combination of CT and 18FDG-positron emission tomography in non-small-cell lung cancer. Int. J. Radiat. Oncol. Biol. Phys. 67: 709-719.

Guckenberger M, Wilbert J, Richter A, Baier K, et al. (2011). Potential of adaptive radiotherapy to escalate the radiation dose in combined radiochemotherapy for locally advanced non-small cell lung cancer. Int. J. Radiat. Oncol. Biol. Phys. 79: 901-908.

Hansen O, Paarup H, Sorensen P, Hansen KH, et al. (2005). Curative radiotherapy of local advanced non-small-cell lung cancer. Eight years of experience from Odense. Ugeskr. Laeger 167: 3497-3502.

Kupelian PA, Ramsey C, Meeks SL, Willoughby TR, et al. (2005). Serial megavoltage CT imaging during external beam radiotherapy for non-small-cell lung cancer: observations on tumor regression during treatment. Int. J. Radiat. Oncol. Biol. Phys. 63: 1024-1028.

O’Brien K, Cokkinides V, Jemal A, Cardinez CJ, et al. (2003). Cancer statistics for Hispanics, 2003. CA Cancer J. Clin. 53: 208-226.

Perez CA, Stanley K, Rubin P, Kramer S, et al. (1980). A prospective randomized study of various irradiation doses and fractionation schedules in the treatment of inoperable non-oat-cell carcinoma of the lung. Preliminary report by the Radiation Therapy Oncology Group. Cancer 45: 2744-2753.

Siker ML, Tome WA and Mehta MP (2006). Tumor volume changes on serial imaging with megavoltage CT for nonsmall-cell lung cancer during intensity-modulated radiotherapy: how reliable, consistent, and meaningful is the effect? Int. J. Radiat. Oncol. Biol. Phys. 66: 135-141.

Sim S, Rosenzweig KE, Schindelheim R, Ng KK, et al. (2001). Induction chemotherapy plus three-dimensional conformal radiation therapy in the definitive treatment of locally advanced non-small-cell lung cancer. Int. J. Radiat. Oncol. Biol. Phys. 51: 660-665.

van Zwienen M, van Beek S, Belderbos J, van Kranen S, et al. (2008). Anatomical changes during radiotherapy of lung cancer patients. Int. J. Radiat. Oncol. Biol. Phys. 72: S111.

Wang YX, He CX, Qu BL, Xu SP, et al. (2006). Three-dimensional conformal radiation therapy for elderly non-small cell lung cancer patients. Chin. J. Radiat. Oncol. 15: 7-9.

Woodford C, Yartsev S, Dar AR, Bauman G, et al. (2007). Adaptive radiotherapy planning on decreasing gross tumor volumes as seen on megavoltage computed tomography images. Int. J. Radiat. Oncol. Biol. Phys. 69: 1316-1322.

Wu KL, Jiang GL, Liao Y, Qian H, et al. (2003). Three-dimensional conformal radiation therapy for non-small-cell lung cancer: a phase I/II dose escalation clinical trial. Int. J. Radiat. Oncol. Biol. Phys. 57: 1336-1344. 